\title{
BMJ Open Interventions to prevent or reduce the level of frailty in community-dwelling older adults: a protocol for a scoping review of the literature and international policies
}

\author{
Martine T E Puts, ${ }^{1}$ Samar Toubasi, ${ }^{1}$ Esther Atkinson, ${ }^{2}$ Ana Patricia Ayala, ${ }^{3}$ \\ Melissa Andrew, ${ }^{4}$ Maureen C Ashe, ${ }^{5}$ Howard Bergman, ${ }^{6}$ Jenny Ploeg, ${ }^{7}$ \\ Katherine S McGilton ${ }^{1,8}$
}

To cite: Puts MTE,

Toubasi S, Atkinson E, et al. Interventions to prevent or reduce the level of frailty in community-dwelling older adults: a protocol for a scoping review of the literature and international policies. BMJ Open 2016;6: e010959. doi:10.1136/ bmjopen-2015-010959

- Prepublication history and additional material is available. To view please visit the journal (http://dx.doi.org/ 10.1136/bmjopen-2015010959)

Received 22 December 2015 Accepted 11 February 2016

CrossMark

For numbered affiliations see end of article.

Correspondence to Dr Martine TE Puts; martine.puts@utoronto.ca

\section{ABSTRACT}

Introduction: With ageing comes increased vulnerability such that older adults' ability to recover from acute illnesses, fall-related injuries and other stresses related to the physical ageing processes declines. This increased vulnerability, also known as frailty, is common in older adults and associated with increased healthcare service use and adverse health outcomes. Currently, there is no overview of available interventions to prevent or reduce the level of frailty (as defined by study's authors) which will help healthcare providers in community settings caring for older adults. We will address this gap by reviewing interventions and international polices that are designed to prevent or reduce the level of frailty in community-dwelling older adults.

Methods and analysis: We will conduct a scoping review using the updated guidelines of Arksey and O'Malley to systematically search the peer-reviewed journal articles to identify interventions that aimed to prevent or reduce the level of frailty. We will search grey literature for international policies. The 6 -stage scoping review model involves: (1) identifying the research question; (2) identifying relevant studies; (3) selecting studies; (4) charting the data; (5) collating, summarising and reporting the results and (6) consulting with key stakeholders.

Ethics and dissemination: Our scoping review will use robust methodology to search for available interventions focused on preventing or reducing the level of frailty in community-dwelling older adults. We will consult with stakeholders to find out whether they find the frailty interventions/policies useful and to identify the barriers and facilitators to their implementation in Canada. We will disseminate our findings to relevant stakeholders at local, national and international levels by presenting at relevant meetings and publishing the findings. Our review will identify gaps in research and provide healthcare providers and policymakers with an overview of interventions that can be implemented to prevent or postpone frailty.

\section{Strengths and limitations of this study}

- Systematic summarisation of the evidence will identify the evidence available from qualitative and quantitative studies and policies; it will also identify gaps in current evidence on interventions aimed at preventing or reducing the level of frailty in community-dwelling older adults.

- We will include all studies published in any language that have measured the level of frailty preintervention and postintervention.

- Although many intervention studies have focused on the frail elderly, there has been no review of interventions that used level of frailty as an outcome, making it impossible to compare different study populations and interventions.

- The review will help inform policies to facilitate acquisition or implementation of resources needed by frail older adults.

\section{INTRODUCTION}

As in most countries, Canada's population is ageing-in 2013, $15 \%$ of the population was aged 65 and over, and this is expected to increase to $27 \%$ by $2063 .{ }^{1}$ As older adults age, they are more vulnerable to poor health outcomes and are less able to recover from an acute stress as a result of physiological changes, that is, declines in muscle mass and kidney, lung and cardiovascular organ functioning. This increased vulnerability is also known as frailty. ${ }^{2}{ }^{3}$ Frail older adults are more at risk for adverse health outcomes, such as falls, mobility decline, hospitalisation, institutionalisation and death. ${ }^{2}{ }^{4-24}$ There is no consensus on the definition of frailty, but it is consistently seen as a multidimensional concept with various indicators such as weight loss, lack of physical activity and lack 
of strength. ${ }^{25-27}$ There are also multiple frailty measurement tools available. Despite the lack of consensus on definition and which indicators and measurement tools to use, frail older adults are seen across all sectors of healthcare: primary care; most acute care specialties (emergency medicine, surgery, cardiology, oncology, nephrology, endocrinology, neurology and critical care); and long-term care. ${ }^{4} 12 \quad 2425$ 27-63 Although the older adult population is heterogeneous across the continuum from very fit to frail, frailty presents unique challenges that significantly affect older adults, their families and society.

Research highlights that frailty is potentially reversible. ${ }^{64}$ Therefore, identification and management of frailty to prevent, reduce and postpone adverse health consequences for older adults and their families is warranted. Despite the extensive body of research focusing on indicators and outcomes of frailty, only six reviews of frailty and prevention/treatment interventions have been published: two reviews included randomised controlled intervention trials for patients in the cardiac care setting only, ${ }^{65}{ }^{66}$ three expert reviews examined predominantly pharmaceutical interventions but did not use a systematic approach to searching and analysing the evidence, ${ }^{67-69}$ and one review included randomised physical interventions/exercise trials focusing on physical performance outcome measures only. ${ }^{70}$ Currently, two review protocols in progress are listed on the Prospero/ Cochrane Systematic review database; one focuses exclusively on mobility interventions in frail and prefrail populations including (randomised) controlled trials only, ${ }^{71}$ and the other focuses on home-based health promotion delivered by healthcare professionals for older adults with early frailty ${ }^{72}$ and includes studies published in English only. Thus, to date, there has not been a comprehensive review of interventions that can prevent or reduce the level of frailty in community-dwelling older adults that can be implemented by either primary healthcare providers and/or older adults and/or their families/caregivers in their home or in the community focusing on outcomes that are important to older adults such as level of frailty, functional status, quality of life and the ability to remain in their home (eg, avoid a transition to an alternative care setting). In addition, in our review, we will search for international policies that are in use to prevent or reduce the level of frailty; this element has not been included in previous and currently ongoing reviews. Owing to the absence of a comprehensive review on frailty interventions, older adults and their families, and healthcare professionals have little guidance to prevent or reduce the level of frailty. For healthcare providers working in primary healthcare settings, a comprehensive review of interventions they can deliver as well as single domain and multidomain frailty interventions that older adults and/or their caregivers can participate in is a next important step. We have chosen to use a scoping review methodology, so as to include all types of studies and policies that have targeted the level of frailty of the older adult. The review will summarise the available evidence of what has been done to prevent or to reduce the level of frailty as well as the impact of frailty interventions and policies. This type of review also allows us to include consultations with key stakeholders to identify gaps in the evidence and research that need to be addressed in future research.

Interventions could prevent or delay negative health outcomes of frailty for community-dwelling older adults with impacts on family members/caregivers, healthcare providers and the healthcare system. Further, early management of frailty may contribute to improved quality of life for the older adult and their family members/caregivers. As frailty often cannot be averted in persons growing old, its management may lead to better outcomes and delay institutionalisation. Therefore, our research question for this scoping review is: Which interventions and policies are effective in preventing or reducing the level of frailty in community-dwelling older adults? Only studies that have measured frailty with an explicit operational definition outlined by the study's authors preintervention and postintervention will be included. The key outcome is the level of frailty and additional outcomes may also include functional status, quality of life, utilisation of healthcare and transitions in care settings. The purpose of the review is to examine the literature for evidence that will inform the design and implementation of interventions to prevent or reduce the level of frailty. The outcome of the review will include a summary of available interventions and effective policies and an identification of trials in progress and gaps in research. This will help inform policies to facilitate acquisition or implementation of resources needed by frail older adults

\section{METHODS AND ANALYSIS \\ Methodology}

We will use the scoping review methodological framework described by Arksey and O'Malley ${ }^{73}$ with refinements to this framework as described by Levac et $a l^{74}$ Colquhoun et $a l^{75}$ and Daudt $e t a l^{76}$ and following the PRISMA-P statement. ${ }^{77}$ This six-stage model involves: (1) identifying the research question (listed above); (2) identifying relevant studies (search methods used); (3) selecting studies; (4) charting the data; (5) collating, summarising and reporting the results and (6) consulting with key stakeholders. The refinements to the original framework include establishing a clear research question, purpose and outcome of the scoping review; assembling a team with content and methodological expertise; searching the literature using an iterative process with inclusion and exclusion criteria; using at least two reviewers to independently review abstracts and full-text papers, with a consensus procedure in case of a disagreement; developing a data abstraction form where two researchers abstract the data independently; 
conducting a quality assessment of included papers; and performing an analysis which includes a descriptive numerical summary of papers as well as a qualitative thematic analysis. Finally, consultation with key stakeholders is a required step in a scoping review and is not optional.

\section{Search methods}

It is recommended that searches for scoping reviews are as comprehensive as possible to identify all possible studies. $^{73-76}$ Our inclusion criteria are: all types of original studies (quantitative, qualitative or mixed-methods studies) or reports studying an intervention to prevent or to reduce the level of frailty in community-dwelling patients aged 65 and over. Studies that included a wider age range are eligible as long as the mean/median age of the study population is aged 65 years and older or if they have included a subgroup analysis for the population aged 65 years and over. Included studies will provide a measure of frailty (as defined by study's authors) before and after the implemented intervention. Grey literature (reports by healthcare/health policy organisations and Opengrey) that has information on the effect of the interventions to prevent or reduce frailty is eligible. Exclusion criteria include expert opinions and editorials that do not include original data.

The search will cover studies published from January 2000 to September 2015 using key words and Medical Subject Headings (MeSH) terms such as frail elderly, frailty AND interventions, evaluation study, multicentre study, randomised trial, intervention study, AND Aged, 65 and over, senior. We will restrict the search to recent studies (2000-2015) to ensure that identified interventions will be relevant to current clinical practice. Searches (including grey literature) will be performed with no language restrictions and carried out by two librarians on our team with expertise in review methodology (EA and APA). See online supplementary appendix A for the MEDLINE search strategy. If studies are identified in languages other than those mastered by the research team, we will contact the authors to complete the data abstraction and quality assessment form.

We will search the Cochrane Central Register of Controlled Trials (CENTRAL), Cochrane Effective Practice and Organisation of Care Group (EPOC), MEDLINE, EMBASE, Cumulative Index to Nursing and Allied Health (CINAHL), Allied and Complementary Medicine (AMED), Psych INFO, Ageline, Sociological Abstracts, Web of Science, Applied Social Sciences Index and Abstracts (ASSIA), Database of Abstracts of Reviews of Effects (DARE), Health Technology Assessment (Canadian Search Interface) databases, and reference lists of included studies. We will also search conference proceedings of all international geriatrics and gerontology societies meetings. We will contact experts in the field to retrieve any unpublished studies. We will examine trial registries (clinicaltrials.gov, the WHO's International Clinical Trials Registry Platform (ICTRP),
European Union Clinical Trials Register (EU-CTR), Australia and New Zealand's Trial Registry (ANZCTR) and Japan's Trial Registry (UMIN-CTR) for potential studies that are in progress. We will also search for grey literature and international policies using Google, Yahoo and Opengrey.

\section{Study selection}

We will include studies through a two-step process. First, abstracts will be scanned by two independent reviewers per abstract (MTEP, ST and KSM). Then all potentially relevant full articles will be retrieved for inclusion consideration by the same reviewers. Data will be abstracted by two independent reviewers (MTEP, ST and KSM). Bibliographic information will be downloaded in Reference Manager Software. In case of disagreement between the two reviewers, the other team members will contribute to a decision. During this project, we will organise monthly teleconferences to discuss the progress and findings. Any article on which the review team cannot reach consensus will be emailed to all for discussion during the monthly team teleconference. Levac et $a l^{74}$ and Daudt et $a l^{76}$ recommend using a systematic review approach to study selection for scoping reviews to enhance rigour of the review, and thus we will use this approach.

\section{Data abstraction}

We will use standardised data collection forms (Microsoft Excel sheets for the data on the studies and interventions as well as quality assessment of the included studies) developed by the research team. Data will be abstracted by each of the two reviewers independently and compared. The information abstracted will include the following details: country or origin, characteristics of the study population (ie, ethnicity, age, sex, educational level and presence of cognitive impairment), study design, response rates, follow-up and retention rates. We will describe how representative the study population is compared with the general population for each study if the data are available. The data abstracted will further include the definition of frailty (which may include physical, social and cognitive domains); frailty assessment used; levels of frailty of study participants preintervention and postintervention; intervention (description of the type of intervention delivered, intervention allocation, who delivered the intervention, intervention duration, intervention fidelity and randomisation methods); effects of the intervention on outcomes (list of outcomes and if available sensitivity of outcomes); analyses used to examine outcomes (results of the intervention/policy); and quality of the study (see Quality assessment section).

\section{Quality assessment}

As recommended by Daudt et $a l^{76}{ }^{76}$ we will assess the quality of the included study using the Mixed Methods Appraisal Tool (MMAT), ${ }^{78} 79$ a validated tool that allows 
inclusion of qualitative and quantitative studies in a review. The MMAT has been designed to include all types of studies, but for each of the five types of study design in the MMAT (qualitative, mixed methods, quantitative randomised trials, quantitative non-randomised and quantitative descriptive), there are different quality assessment criteria. The MMAT is not intended to exclude studies based on the quality assessment result. We will thus not exclude studies based on the quality assessment as we want to provide a comprehensive overview of the available evidence.

\section{Data analysis}

We will summarise the results using a narrative descriptive synthesising approach. ${ }^{73-76}$ We anticipate that there will be differences in frailty definitions and different outcome measures of intervention effectiveness that will preclude conducting a meta-analysis. Data collected will be summarised by tabulating all interventions and their impact on outcomes that were studied. We will also summarise the interventions' effect for those delivered by healthcare providers and those carried out by older adults and/or their family members/caregivers. We will also summarise the interventions' effect for those delivered in the home versus other community settings (family physician's office, community centre, other community location). We will calculate effect sizes and numbers needed to treat where possible. Policies in use to target the level of frailty in community-dwelling older adults will be described and summarised separately from the studies identified through the literature searches. By summarising the evidence base and critically appraising the studies, we will be able to identify gaps in the current evidence base and where new studies are needed. We will also summarise studies currently in progress, so that other researchers developing new interventions targeting frailty can consult these ongoing studies and methods used.

\section{Consultation}

Arksey and O'Malley ${ }^{73}$ have said consultation is optional, but Levac et $a l^{74}$ and Daudt $e t a l^{76}$ disagree. Our research team includes several healthcare providers from different specialties who will be involved at all steps of this study. We will organise a stakeholder meeting to which we will invite local, provincial and national community care organisations, senior organisations, primary care organisations and Ontario Ministry of Health and Long-Term Care representatives to provide feedback on the findings and to develop next steps in research and practice. The feedback from the stakeholder meeting ${ }^{80-82}$ and the results of the scoping review will be combined to clearly indicate the available evidence, gaps in research and future research priorities for this population.

\section{ETHICS AND DISSEMINATION}

This scoping review aims to identify all interventions that have targeted to prevent or reduce the level of frailty in community-dwelling older adults. In addition to peerreviewed literature, we will also include grey literature and international policies using standardised scoping review methodologies. We anticipate our scoping review will identify the gaps in research for preventing and managing frailty in older adults. All data in this project will be gathered through searches of literature databases and policies available online and no personal (health) information will be collected in the context of this project; thus approval from a research ethics committee is not required.

With the ageing of the population, there will be an increase in frail older adults. Frailty is associated with adverse health outcomes for older adults and increased healthcare utilisation. Research has shown that frailty is potentially reversible in early stages. Therefore, it is important to prevent and reduce the level of frailty in the community and primary healthcare settings, to avoid or delay adverse health outcomes for the older adult and their family members to improve their quality of life, and to reduce care transitions and associated costs of unnecessary use of health services. The review, with the overview of which interventions and policies are effective, will assist healthcare providers and health policymakers to select interventions relevant for their clinical practice, and advocate for these to be implemented in their organisations. Health policymakers will be informed of the findings at our stakeholder meetings and we will disseminate our findings to other local, national and international stakeholders by presenting the findings at relevant meetings and by publishing our findings.

\section{CONCLUSION}

The best interventions and polices to prevent or reduce the level of frailty to maintain functional status, quality of life, reduce healthcare costs and to enable this patient population to continue living at home are currently unknown. Most older adults want to remain at home as they age, so it is important to understand the evidence that exists, so that practitioners and policy decision makers are able to ensure resources are directly wisely to prevent and reduce the level of frailty.

\section{Author affiliations}

${ }^{1}$ Lawrence S. Bloomberg Faculty of Nursing, University of Toronto, Toronto, Ontario, Canada

${ }^{2}$ Institute for the History and Philosophy of Science and Technology, University of Toronto, Toronto, Ontario, Canada

${ }^{3}$ Gerstein Information Science Centre, University of Toronto Libraries, University of Toronto, Toronto, Ontario, Canada

${ }^{4}$ Division of Geriatric Medicine, Dalhousie University, Halifax, Nova Scotia,

Canada

${ }^{5}$ Department of Family Medicine, University of British Columbia, Vancouver, British Columbia, Canada

${ }^{6}$ Department of Family Medicine, McGill University, Montreal, Quebec, Canada ${ }^{7}$ School of Nursing, McMaster University, Hamilton, Ontario, Canada

${ }^{8}$ Toronto Rehabilitation Institute, University Health Network, Toronto, Ontario, Canada 
Twitter Follow Ana Patricia Ayala at @apayala

Contributors All authors contributed towards the study design. MTEP, MA, MCA, APA, JP, HB and KSM obtained study funding. MTEP and ST drafted the protocol. EA and APA designed the initial search strategy for protocol submission. All authors have read and approved the final protocol. MTEP is the guarantor of the review.

Funding This work was supported by Technology Evaluation in the Elderly Network (TVN) grant FRA2015-A-07. MTEP is supported by a Canadian Institutes of Health Research New Investigator Award.

Competing interests None declared.

Provenance and peer review Not commissioned; externally peer reviewed.

Open Access This is an Open Access article distributed in accordance with the Creative Commons Attribution Non Commercial (CC BY-NC 4.0) license, which permits others to distribute, remix, adapt, build upon this work noncommercially, and license their derivative works on different terms, provided the original work is properly cited and the use is non-commercial. See: http:// creativecommons.org/licenses/by-nc/4.0/

\section{REFERENCES}

1. Statistics Canada. Growth of the Canadian population from 2013 to 2063. 2015. http://www.statcan.gc.ca/pub/91-520-x/91-520x2014001-eng.htm

2. Fried LP, Tangen CM, Walston J, et al. Frailty in older adults: evidence for a phenotype. J Gerontol A Biol Sci Med Sci 2001;56: M146-56.

3. Walston J, Fried LP. Frailty and the older man. Med Clin North Am 1999;83:1173-94.

4. Ambler GK, Brooks DE, Al Zuhir N, et al. Effect of frailty on shortand mid-term outcomes in vascular surgical patients. Br J Surg 2015;102:638-45.

5. Armstrong JJ, Stolee P, Hirdes JP, et al. Examining three frailty conceptualizations in their ability to predict negative outcomes for home-care clients. Age Ageing 2010;39:755-8.

6. Au A, Puts MT, Fletcher JD, et al. Frailty markers predicting emergency department visits in a community-dwelling sample of vulnerable seniors in Montreal. Can J Aging 2011;30:647-55.

7. Basic D, Shanley C. Frailty in an older inpatient population: using the clinical frailty scale to predict patient outcomes. J Aging Health 2015;27:670-85.

8. Beggs T, Sepehri A, Szwajcer A, et al. Frailty and perioperative outcomes: a narrative review. Can J Anaesth 2015;62:143-57.

9. Bergman $\mathrm{H}$, Ferrucci L, Guralnik J, et al. Frailty: an emerging research and clinical paradigm-issues and controversies. $J$ Gerontol A Biol Sci Med Sci 2007;62:731-7.

10. Courtney-Brooks M, Tellawi AR, Scalici J, et al. Frailty: an outcome predictor for elderly gynecologic oncology patients. Gynecol Oncol 2012;126:20-4.

11. Drubbel I, de Wit NJ, Bleijenberg N, et al. Prediction of adverse health outcomes in older people using a frailty index based on routine primary care data. J Gerontol A Biol Sci Med Sci 2013;68:301-8.

12. Dwyer JG, Reynoso JF, Seevers GA, et al. Assessing preoperative frailty utilizing validated geriatric mortality calculators and their association with postoperative hip fracture mortality risk. Geriatr Orthop Surg Rehabil 2014;5:109-15.

13. Gobbens RJ, van Assen MA, Luijkx KG, et al. The predictive validity of the Tilburg frailty indicator: disability, health care utilization, and quality of life in a population at risk. Gerontologist 2012;52:619-31.

14. Klein BE, Klein R, Knudtson MD, et al. Frailty, morbidity and survival. Arch Gerontol Geriatr 2005;41:141-9.

15. Kristjansson SR, Ronning B, Hurria A, et al. A comparison of two pre-operative frailty measures in older surgical cancer patients. J Geriatr Oncol 2012;3:1-7.

16. Ommundsen $\mathrm{N}$, Wyller TB, Nesbakken A, et al. Frailty is an independent predictor of survival in older patients with colorectal cancer. Oncologist 2014;19:1268-75.

17. Puts MT, Monette J, Girre V, et al. Are frailty markers useful for predicting treatment toxicity and mortality in older newly diagnosed cancer patients? Results from a prospective pilot study. Crit Rev Oncol Hematol 2011;78:138-49.

18. Puts MT, Monette J, Girre V, et al. Does frailty predict hospitalization, emergency department visits, and visits to the general practitioner in older newly-diagnosed cancer patients?
Results of a prospective pilot study. Crit Rev Oncol Hematol 2010;76:142-51.

19. Puts MT, Lips P, Deeg DJ. Sex differences in the risk of frailty for mortality independent of disability and chronic diseases. J Am Geriatr Soc 2005;53:40-7.

20. Robinson TN, Wu DS, Stiegmann GV, et al. Frailty predicts increased hospital and six-month healthcare cost following colorectal surgery in older adults. Am J Surg 2011;202:511-14.

21. Shamliyan T, Talley KM, Ramakrishnan R, et al. Association of frailty with survival: a systematic literature review. Ageing Res Rev 2013;12:719-36.

22. Tan KY, Kawamura YJ, Tokomitsu A, et al. Assessment for frailty is useful for predicting morbidity in elderly patients undergoing colorectal cancer resection whose comorbidities are already optimized. Am J Surg 2012;204:139-43.

23. Uppal S, Igwe E, Rice LW, et al. Frailty index predicts severe complications in gynecologic oncology patients. Gynecol Oncol 2015;137:98-101.

24. Wallis SJ, Wall J, Biram RW, et al. Association of the clinical frailty scale with hospital outcomes. QJM 2015;108:943-9.

25. Clegg A, Rogers L, Young J. Diagnostic test accuracy of simple instruments for identifying frailty in community-dwelling older people: a systematic review. Age Ageing 2015;44:148-52.

26. Handforth $C$, Clegg A, Young $C$, et al. The prevalence and outcomes of frailty in older cancer patients: a systematic review. Ann Oncol 2015;26:1091-101.

27. Turner G, Clegg A. Best practice guidelines for the management of frailty: a British Geriatrics Society, Age UK and Royal College of General Practitioners report. Age Ageing 2014;43:744-7.

28. Lee L, Heckman G, Molnar FJ. Frailty: identifying elderly patients at high risk of poor outcomes. Can Fam Physician 2015;61:227-31.

29. Lacas A, Rockwood K. Frailty in primary care: a review of its conceptualization and implications for practice. BMC Med 2012;10:4

30. Pialoux T, Goyard J, Lesourd B. Screening tools for frailty in primary health care: a systematic review. Geriatr Gerontol Int 2012;12:189-97.

31. Hoogendijk EO, van der Horst HE, Deeg DJ, et al. The identification of frail older adults in primary care: comparing the accuracy of five simple instruments. Age Ageing 2013;42:262-5.

32. Bagshaw SM, Stelfox HT, Johnson JA, et al. Long-term association between frailty and health-related quality of life among survivors of critical illness: a prospective multicenter cohort study. Crit Care Med 2015;43:973-82.

33. Fairhall N, Kurrle SE, Sherrington C, et al. Effectiveness of a multifactorial intervention on preventing development of frailty in pre-frail older people: study protocol for a randomised controlled trial. BMJ Open 2015;5:e007091.

34. Lam M, Jassal SV. The concept of frailty in geriatric chronic kidney disease (CKD) patients. Blood Purif 2015;39:50-4.

35. Hii TB, Lainchbury JG, Bridgman PG. Frailty in acute cardiology comparison of a quick clinical assessment against a validated frailty assessment tool. Heart Lung Circ 2015;24:551-6.

36. Romero-Ortuno R, Soraghan C. A Frailty Instrument for primary care for those aged 75 years or more: findings from the Survey of Health, Ageing and Retirement in Europe, a longitudinal population-based cohort study (SHARE-FI75+). BMJ Open 2014;4:e006645.

37. Ness KK, Armstrong GT, Kundu M, et al. Frailty in childhood cancer survivors. Cancer 2015;121:1540-7.

38. Milte R, Crotty M. Musculoskeletal health, frailty and functional decline. Best Pract Res Clin Rheumatol 2014;28:395-410.

39. Cosco TD, Armstrong JJ, Stephan BC, et al. Successful aging and frailty: mutually exclusive paradigms or two ends of a shared continuum? Can Geriatr J 2015;18:35-6.

40. Lowry KA, Vallejo AN, Studenski SA. Successful aging as a continuum of functional independence: lessons from physical disability models of aging. Aging Dis 2012;3:5-15.

41. Schoufour JD, Echteld MA, Evenhuis HM. [Frailty in people with intellectual disabilities: operationalization, risks and detection]. Tijdschr Gerontol Geriatr 2015;46:92-103.

42. Tapper EB, Finkelstein D, Mittleman MA, et al. Standard assessments of frailty are validated predictors of mortality in hospitalized patients with cirrhosis. Hepatology 2015;62:584-90.

43. Hoogendijk EO, van Kan GA, Guyonnet S, et al. Operationalization of a frailty index using routine data from the Toulouse Frailty Clinic. Geriatr Gerontol Int 2015;15:519-20.

44. Uchmanowicz I, Lisiak M, Wontor R, et al. Frailty in patients with acute coronary syndrome: comparison between tools for comprehensive geriatric assessment and the Tilburg Frailty Indicator. Clin Interv Aging 2015;10:521-9.

45. Flint K. Which came first, the frailty or the heart disease? Exploring the vicious cycle. J Am Coll Cardiol 2015;65:984-6. 
46. Babayan RK. Re: a prospective study examining the association between preoperative frailty and postoperative complications in patients undergoing minimally invasive surgery. $J$ Urol 2015;193:907-8.

47. van Hateren $\mathrm{KJ}$, Hendriks $\mathrm{SH}$, Groenier $\mathrm{KH}$, et al. Frailty and the relationship between blood pressure and mortality in elderly patients with type 2 diabetes (Zwolle Outpatient Diabetes project Integrating Available Care-34). J Hypertens 2015;33:1162-6.

48. Arai $\mathrm{H}$, Ouchi $\mathrm{Y}$, Yokode $\mathrm{M}$, et al. Toward the realization of a better aged society: messages from gerontology and geriatrics. Geriatr Gerontol Int 2012;12:16-22.

49. Bansal N, Dhaliwal R, Weinstock RS. Management of diabetes in the elderly. Med Clin North Am 2015;99:351-77.

50. Musso CG, Vilas M, Onuigbo M. Nephroprevention in the oldest old with chronic kidney disease: special considerations. World J Nephrol 2015;4:1-5

51. Armstrong JJ, Andrew MK, Mitnitski A, et al. Social vulnerability and survival across levels of frailty in the Honolulu-Asia Aging Study. Age Ageing 2015;44:709-12.

52. Bras L, Peters TT, Wedman J, et al. Predictive value of the Groningen Frailty Indicator for treatment outcomes in elderly patients after head and neck, or skin cancer surgery in a retrospective cohort. Clin Otolaryngol 2015;40:474-82.

53. Maxwell CA, Mion LC, Mukherjee K, et al. Feasibility of screening for preinjury frailty in hospitalized injured older adults. J Trauma Acute Care Surg 2015;78:844-51.

54. Leng SX, Margolick JB. Understanding frailty, aging, and inflammation in HIV infection. Curr HIV/AIDS Rep 2015;12:25-32.

55. Stewart R. Do risk factors for cardiovascular disease also increase the risk of frailty? Heart 2015;101:582-3.

56. Abbatecola AM, Paolisso G, Sinclair AJ. Treating diabetes mellitus in older and oldest old patients. Curr Pharm Des 2015;21:1665-71.

57. Chen CC, Lin MT, Liang JT, et al. Pre-surgical geriatric syndromes, frailty, and risks for postoperative delirium in older patients undergoing gastrointestinal surgery: prevalence and red flags. $J$ Gastrointest Surg 2015;19:927-34.

58. Romanowski KS, Barsun A, Pamlieri TL, et al. Frailty score on admission predicts outcomes in elderly burn injury. J Burn Care Res 2015;36:1-6

59. Goldstein J, Hubbard RE, Moorhouse P, et al. The validation of a care partner-derived frailty index based upon comprehensive geriatric assessment (CP-FI-CGA) in emergency medical services and geriatric ambulatory care. Age Ageing 2015;44:327-30.

60. McAdams-DeMarco MA, Law A, King E, et al. Frailty and mortality in kidney transplant recipients. Am J Transplant 2015;15:149-54.

61. Haveman-Gould $B$, Lyons K. Assessing frailty in surgical candidates. JAAPA 2014:27:45-9.

62. Rowe R, lqbal J, Murali-Krishnan R, et al. Role of frailty assessment in patients undergoing cardiac interventions. Open Heart 2014;1: e000033.

63. Thorsteinsdottir B, Swetz KM, Tilburt JC. Dialysis in the frail elderlya current ethical problem, an impending ethical crisis. J Gen Intern Med 2013;28:1511-16.

64. Ng TP, Feng L, Nyunt MS, et al. Nutritional, physical, cognitive, and combination interventions and frailty reversal among older adults: a randomized controlled trial. Am J Med 2015;128:1225-36.
65. Bibas L, Levi M, Bendayan M, et al. Therapeutic interventions for frail elderly patients: part I. Published randomized trials. Prog Cardiovasc Dis 2014;57:134-43.

66. Bendayan M, Bibas L, Levi M, et al. Therapeutic interventions for frail elderly patients: part II. Ongoing and unpublished randomized trials. Prog Cardiovasc Dis 2014;57:144-51.

67. Laosa O, Alonso C, Castro M, et al. Pharmaceutical interventions for frailty and sarcopenia. Curr Pharm Des 2014;20:3068-82.

68. Jeffery CA, Shum DW, Hubbard RE. Emerging drug therapies for frailty. Maturitas 2013;74:21-5.

69. Rolland $\mathrm{Y}$, Dupuy C, Abellan van Kan G, et al. Treatment strategies for sarcopenia and frailty. Med Clin North Am 2011;95:427-38, ix.

70. Giné-Garriga M, Roqué-Figuls $\mathrm{M}$, Coll-Planas L, et al. Physical exercise interventions for improving performance-based measures of physical function in community-dwelling, frail older adults: a systematic review and meta-analysis. Arch Phys Med Rehabil 2014;95:753-69.

71. Fairhall N, Sherrington C, Cameron I. Mobility training for increasing mobility and functioning in older people with frailty. Cochrane Library, 2013. http://onlinelibrary.wiley.com/doi/10.1002/14651858. CD010494/pdf.

72. Walters K, Jovicic A, Kharicha K, et al. Home-based health promotion for older people with early frailty. 2014. http://www.crd. york.ac.uk/PROSPERO/display_record.asp?ID=CRD42014010370

73. Arksey H, O'Malley L. Scoping reviews: towards a methodological framework. Int J Soc Res Methodol 2005;8:19-32.

74. Levac D, Colquhoun H, O'Brien KK. Scoping studies: advancing the methodology. Implement Sci 2010;5:69.

75. Colquhoun $\mathrm{HL}$, Levac D, O'Brien KK, et al. Scoping reviews: time for clarity in definition, methods, and reporting. J Clin Epidemiol 2014:67:1291-4.

76. Daudt HM, van Mossel C, Scott SJ. Enhancing the scoping study methodology: a large, inter-professional team's experience with Arksey and O'Malley's framework. BMC Med Res Methodol 2013;13:48.

77. Shamseer L, Moher D, Clarke M, et al. Preferred reporting items for systematic review and meta-analysis protocols (PRISMA-P) 2015: elaboration and explanation. BMJ 2015;349:g7647.

78. Souto RQ, Khanassov V, Hong QN, et al. Systematic mixed studies reviews: updating results on the reliability and efficiency of the Mixed Methods Appraisal Tool. Int J Nurs Stud 2015;52:500-1.

79. Pace R, Pluye P, Bartlett G, et al. Testing the reliability and efficiency of the pilot Mixed Methods Appraisal Tool (MMAT) for systematic mixed studies review. Int J Nurs Stud 2012:49:47-53.

80. Concannon TW, Fuster M, Saunders T, et al. A systematic review of stakeholder engagement in comparative effectiveness and patient-centered outcomes research. J Gen Intern Med 2014;29:1692-701.

81. Cottrell E, Whitlock E, Kato E, et al. Defining the benefits of stakeholder engagement in systematic reviews. Rockville, MD: Agency for Healthcare Research and Quality (US), 2014.

82. O'Haire C, McPheeters M, Nakamoto E, et al. Engaging stakeholders to identify and prioritize future research needs. Rockville, MD: Agency for Healthcare Research and Quality (US), 2011. 